\title{
Trigonometric shock waves for the Kaup-Boussinesq system
}

\author{
Sergey K. Ivanov ${ }^{1,2}$ and Anatoly M. Kamchatnov ${ }^{1,2}$ \\ ${ }^{1}$ Moscow Institute of Physics and Technology, Institutsky lane 9, Dolgoprudny, Moscow region, 141700, Russia \\ ${ }^{2}$ Institute of Spectroscopy, Russian Academy of Sciences, Troitsk, Moscow, 108840, Russia
}

\begin{abstract}
We consider the modulationally stable version of the Kaup-Boussinesq system which models propagation of nonlinear waves in various physical systems. It is shown that the Whitham modulation equations for this model have a new type of solutions which describe trigonometric shock waves. In the Gurevich-Pitaevskii problem of evolution of an initial discontinuity these solutions correspond to a non-zero wave excitation on one of the sides of the discontinuity. Our analytical results are confirmed by numerical calculations.
\end{abstract}

\section{INTRODUCTION}

Dispersion in nonlinear systems can dramatically affect wave profile leading to a host of new physical wave structures such as solitons and dispersive shock waves. In particular, it is now well known that in such systems a typical evolution of an initial pulse with a fairly smooth and large initial profile is accompanied by a gradual steepening followed by the wave breaking and formation of dispersive shock wave (DSW). Theoretically, dispersive shock waves, also called undular bores in fluid mechanics applications, are represented as modulated nonlinear periodic waves and then the process of their formation and evolution is described in the Gurevich-Pitaevskii approximation [1] by the Whitham theory of modulations [2] (for reviews see [3, 4]). The original formulation of Gurevich and Pitaevskii approach was applied to description of expanding collisionless shocks (plasma analogs of DSWs) in framework of the Whitham-averaged equations for the integrable Korteweg-de Vries (KdV) equation [5, 6]. Due to universality of the KdV equation, this approach can naturally be applied to many other physical situations and it was extended to many other nonlinear wave equations. For example, when the condition of unidirectional propagation of the $\mathrm{KdV}$ approximation is relaxed, shallow water waves are described by various forms of the Boussinesq equations 7 . The most convenient for our purposes form has been derived by Kaup 8 ; this is the so-called Kaup-Boussinesq (KB) system which is also integrable by the inverse scattering transform method. Periodic solutions of the KB system were obtained in Ref. 9] and the corresponding Gurevich-Pitaevskii theory was extended to the KB case in Refs. $10-12$.

In the applications of Gurevich-Pitaevskii approach to concrete water wave problems, the KB equations with negative dispersion are used. However, the dispersion relation for this kind of equations corresponds to a dynamical instability of small wavelength perturbations over a fluid of constant depth $h_{0}$. There exists another form of the KB system with positive dispersion

$$
\begin{aligned}
& h_{t}+(h u)_{x}-\frac{1}{4} u_{x x x}=0, \\
& u_{t}+u u_{x}+h_{x}=0,
\end{aligned}
$$

where $h$ is the local height of the water layer and $u$ is a local mean flow velocity. For this equation the dispersion relation of linear waves reads

$$
\omega^{2}=h_{0} k^{2}+\frac{1}{4} k^{4}
$$

and the system (1) does not suffer from this kind of deficiency. Moreover, it appears as an approximation to the nonlinear polarization dynamics of a two-component Bose-Einstein condensate [13] as well as to dynamics of magnetization in magnetics with easy-plane anisotropy, so it deserves thorough investigation.

In Ref. 12 the Riemann problem of evolution of initial discontinuities was studied for the system (1). Here we consider the initial states of a different type: we assume that on one side of the initial discontinuity the profiles are represented by periodic solutions rather than by uniform distributions, as it supposed in the standard Riemann problem. This means that our theory describes spreading out of the front of the nonlinear wave excitation along the rarefaction wave. This opens a new route to analytical description of wave structures arising from more complex initial states.

\section{PERIODIC SOLUTIONS AND WHITHAM EQUATIONS}

In this section, we derive the periodic wave solutions of the system (1) and the Whitham equations governing the modulational dynamics. The KB system (1) is completely integrable and it can be represented as a compatibility condition of two linear equations (see [8]) with a free spectral parameter $\lambda$ :

$$
\psi_{x x}=\mathcal{A} \psi, \quad \psi_{t}=-\frac{1}{2} \mathcal{B}_{x} \psi+\mathcal{B} \psi_{x}
$$

where

$$
\mathcal{A}=h-\left(\lambda-\frac{1}{2} u\right)^{2}, \quad \text { and } \quad \mathcal{B}=-\left(\lambda+\frac{1}{2} u\right) .
$$

This permits one to find its periodic solutions and Whitham modulation equations.

Periodic solution can be obtained by the well-known finite-gap integration method (see, e.g., [14). The second order spatial linear differential equation in (3) has two 
basis solutions $\psi_{+}(x, t)$ and $\psi_{-}(x, t)$. Their product $g=$ $\psi_{+} \psi_{-}$satisfies the following third order equation

$$
g_{x x x}-2 \mathcal{A}_{x} g-4 \mathcal{A} g_{x}=0 .
$$

Upon multiplication by $g$, this equation can be integrated once to give

$$
\frac{1}{2} g g_{x x}-\frac{1}{4} g_{x}^{2}-\mathcal{A} g^{2}=P(\lambda)
$$

where the integration constant has been written as $P(\lambda)$ since it can only depend on $\lambda$. The time dependence of $g(x, t)$ is determined by the equation

$$
g_{t}=\mathcal{B} g_{x}-\mathcal{B}_{x} g
$$

We are interested in the one-phase periodic solutions of the system (1). They are distinguished by the condition that $P(\lambda)$ in (6) is a fourth degree polynomial of the form

$$
P(\lambda)=\prod_{i=1}^{4}\left(\lambda-\lambda_{i}\right)=\lambda^{4}-s_{1} \lambda^{3}+s_{2} \lambda^{2}-s_{3} \lambda+s_{4}
$$

Here $s_{i}$ are standard symmetric functions of four zeros $\lambda_{i}$ of the polynomial,

$$
\begin{aligned}
& s_{1}=\sum_{i} \lambda_{i}, \quad s_{2}=\sum_{i<j} \lambda_{i} \lambda_{j}, \quad s_{3}=\sum_{i<j<k} \lambda_{i} \lambda_{j} \lambda_{k} \\
& s_{4}=\lambda_{1} \lambda_{2} \lambda_{3} \lambda_{4} .
\end{aligned}
$$

We shall assume that $\lambda_{i}$ are ordered according to inequalities

$$
\lambda_{1} \leq \lambda_{2} \leq \lambda_{3} \leq \lambda_{4}
$$

Then we find from Eq. (6) that $g(x, t)$ is a first-degree polynomial in $\lambda$,

$$
g(x, t)=\lambda-\mu(x, t)
$$

where $\mu(x, t)$ is connected with $u(x, t)$ and $h(x, t)$ by the relations

$$
\begin{aligned}
u(x, t) & =s_{1}-2 \mu(x, t), \\
h(x, t) & =\frac{1}{4} s_{1}^{2}-s_{2}-2 \mu^{2}(x, t)+s_{1} \mu(x, t),
\end{aligned}
$$

which follow from a comparison of the coefficients of like powers of $\lambda$ on both sides of Eq. (6). The spectral parameter $\lambda$ is arbitrary and on substitution of $\lambda=\mu$ into Eq. 6) we obtain the equation for $\mu$,

$$
\mu_{x}=2 \sqrt{-P(\mu)}
$$

while a similar substitution into Eq. (7) gives

$$
\mu_{t}=-\left(\mu+\frac{1}{2} u\right) \mu_{x}=-\frac{1}{2} s_{1} \mu_{x}
$$

This shows that $\mu$ and hence $h$ and $u$ depend on the variable

$$
\theta=x-\frac{1}{2} s_{1} t
$$

only, so that

$$
V=\frac{1}{2} s_{1}=\frac{1}{2} \sum_{i=1}^{4} \lambda_{i}
$$

is the phase velocity of the nonlinear wave, and $\mu(\theta)$ is determined by the equation

$$
\mu_{\theta}=2 \sqrt{-P(\mu)}
$$

\section{A. Periodic solutions}

The real solution of Eq. 15 corresponds to oscillations of $\mu$ in one of two possible intervals, $\lambda_{1} \leq \mu \leq \lambda_{2}$ or $\lambda_{3} \leq \mu \leq \lambda_{4}$.

- We first consider the periodic solution corresponding to oscillations of $\mu$ in the interval

$$
\lambda_{1} \leq \mu \leq \lambda_{2}
$$

Standard calculation yields, after some algebra, the solution in terms of Jacobi elliptic functions with the initial condition $\mu(0)=\lambda_{1}$ :

$$
\mu(\theta)=\lambda_{2}-\frac{\left(\lambda_{2}-\lambda_{1}\right) \mathrm{cn}^{2}(W, m)}{1+\frac{\lambda_{2}-\lambda_{1}}{\lambda_{4}-\lambda_{2}} \operatorname{sn}^{2}(W, m)},
$$

where $W=\sqrt{\left(\lambda_{3}-\lambda_{1}\right)\left(\lambda_{4}-\lambda_{2}\right)} \theta$ and

$$
m=\frac{\left(\lambda_{2}-\lambda_{1}\right)\left(\lambda_{4}-\lambda_{3}\right)}{\left(\lambda_{3}-\lambda_{1}\right)\left(\lambda_{4}-\lambda_{2}\right)}
$$

Substitution of 17 into 12 gives the corresponding expressions for $u(\theta)$ and $h(\theta)$ for a one-phase periodic nonlinear wave. The wavelength of the wave is equal to

$$
L=\int_{\lambda_{1}}^{\lambda_{2}} \frac{d \mu}{\sqrt{-P(\mu)}}=\frac{2 K(m)}{\sqrt{\left(\lambda_{3}-\lambda_{1}\right)\left(\lambda_{4}-\lambda_{2}\right)}}
$$

where $K(m)$ is the complete elliptic integral of the first kind [15].

The soliton solution corresponds to the limit $\lambda_{3} \rightarrow \lambda_{2}$ $(m \rightarrow 1)$ and in the limit $\lambda_{2} \rightarrow \lambda_{1}$ we get a smallamplitude harmonic wave (see [12]). However, here we are interested in the solution in the form of a trigonometric wave: if $\lambda_{4}=\lambda_{3}$ but $\lambda_{1} \neq \lambda_{2}$, then we have $m=0$ and Eq. 17 reduces to a nonlinear trigonometric wave

$$
\begin{aligned}
\mu(\theta) & =\lambda_{2}-\frac{\left(\lambda_{2}-\lambda_{1}\right) \cos ^{2} W}{1+\frac{\lambda_{2}-\lambda_{1}}{\lambda_{4}-\lambda_{2}} \sin ^{2} W}, \\
W & =\sqrt{\left(\lambda_{4}-\lambda_{1}\right)\left(\lambda_{4}-\lambda_{2}\right)} \theta .
\end{aligned}
$$


If we take the limit $\lambda_{2}-\lambda_{1} \ll \lambda_{4}-\lambda_{1}$ in this solution, then we get the small-amplitude limit

$$
\mu(\theta) \cong \lambda_{2}-\frac{1}{2}\left(\lambda_{2}-\lambda_{1}\right) \cos \left[\left(\lambda_{3}-\lambda_{1}\right) \theta\right] .
$$

On the other hand, if we take here the limit $\lambda_{2} \rightarrow \lambda_{3}=$ $\lambda_{4}$, then the argument of the trigonometric functions becomes small and we can approximate them by the first terms of their series expansions. This corresponds to an algebraic soliton of the form

$$
\mu(\theta)=\lambda_{2}-\frac{\lambda_{2}-\lambda_{1}}{1+\left(\lambda_{2}-\lambda_{1}\right)^{2} \theta^{2}} .
$$

- In a similar way, in the second case,

$$
\lambda_{3} \leq \mu \leq \lambda_{4}
$$

the cnoidal wave solutions are given by the expressions $\left(\mu(0)=\lambda_{4}\right)$

$$
\mu(\theta)=\lambda_{3}+\frac{\left(\lambda_{4}-\lambda_{3}\right) \mathrm{cn}^{2}(W, m)}{1+\frac{\lambda_{4}-\lambda_{3}}{\lambda_{3}-\lambda_{1}} \operatorname{sn}^{2}(W, m)} .
$$

Here the soliton limit can be obtained at $\lambda_{3} \rightarrow \lambda_{2}(m \rightarrow$ 1). In the limit $\lambda_{4} \rightarrow \lambda_{3}$ we get a small-amplitude harmonic wave. In this case, nonlinear trigonometric waves also exists and is attained for $\lambda_{1}=\lambda_{2}$ (but $\lambda_{3} \neq \lambda_{4}$ )

$$
\begin{aligned}
\mu(\theta) & =\lambda_{3}+\frac{\left(\lambda_{4}-\lambda_{3}\right) \cos ^{2} W}{1+\frac{\lambda_{4}-\lambda_{3}}{\lambda_{3}-\lambda_{1}} \sin ^{2} W}, \\
W & =\sqrt{\left(\lambda_{3}-\lambda_{1}\right)\left(\lambda_{4}-\lambda_{1}\right)} \theta .
\end{aligned}
$$

If furthermore $\lambda_{3} \rightarrow \lambda_{1}$, then one gets the algebraic soliton:

$$
\mu(\theta)=\lambda_{1}+\frac{\lambda_{4}-\lambda_{1}}{1+\left(\lambda_{4}-\lambda_{1}\right)^{2} \theta^{2}} .
$$

At last, if we assume that $\lambda_{4}-\lambda_{3} \ll \lambda_{4}-\lambda_{1}$, then we arrive at the small-amplitude limit

$$
\mu(\theta) \cong \lambda_{3}-\frac{1}{2}\left(\lambda_{4}-\lambda_{3}\right) \cos \left[\left(\lambda_{3}-\lambda_{1}\right) \theta\right] .
$$

\section{B. Whitham modulation equations}

Now we shall consider slowly modulated waves. In this case, the parameters $\lambda_{i}(i=1,2,3,4)$ become slowly varying functions of $x$ and $t$ changing little in one period and they can serve as Riemann invariants of modulation equations (see [14]). Evolution of $\lambda_{i}$ is governed by the Whitham equations

$$
\frac{\partial \lambda_{i}}{\partial t}+v_{i} \frac{\partial \lambda_{i}}{\partial x}=0, \quad i=1,2,3,4
$$

The Whitham velocities $v_{i}$ can be computed by means of the formulas

$$
v_{i}\left(\lambda_{1}, \lambda_{2}, \lambda_{3}, \lambda_{4}\right)=\left(1-\frac{L}{\partial_{\lambda_{i}} L} \partial_{\lambda_{i}}\right) V, \quad i=1,2,3,4,
$$

where the phase velocity $V$ and the wavelength $L$ are given by Eqs. (14) and (19). A simple calculation yields the explicit expressions

$$
\begin{aligned}
& v_{1}=\frac{1}{2} \sum_{i=1}^{4} \lambda_{i}-\frac{\left(\lambda_{4}-\lambda_{1}\right)\left(\lambda_{2}-\lambda_{1}\right) K(m)}{\left(\lambda_{4}-\lambda_{1}\right) K(m)-\left(\lambda_{4}-\lambda_{2}\right) E(m)}, \\
& v_{2}=\frac{1}{2} \sum_{i=1}^{4} \lambda_{i}+\frac{\left(\lambda_{3}-\lambda_{2}\right)\left(\lambda_{2}-\lambda_{1}\right) K(m)}{\left(\lambda_{3}-\lambda_{2}\right) K(m)-\left(\lambda_{3}-\lambda_{1}\right) E(m)}, \\
& v_{3}=\frac{1}{2} \sum_{i=1}^{4} \lambda_{i}-\frac{\left(\lambda_{4}-\lambda_{3}\right)\left(\lambda_{3}-\lambda_{2}\right) K(m)}{\left(\lambda_{3}-\lambda_{2}\right) K(m)-\left(\lambda_{4}-\lambda_{2}\right) E(m)}, \\
& v_{4}=\frac{1}{2} \sum_{i=1}^{4} \lambda_{i}+\frac{\left(\lambda_{4}-\lambda_{2}\right)\left(\lambda_{4}-\lambda_{1}\right) K(m)}{\left(\lambda_{4}-\lambda_{1}\right) K(m)-\left(\lambda_{3}-\lambda_{1}\right) E(m)},
\end{aligned}
$$

where $m$ is given by 18$)$ and $K(m)$ and $E(m)$ are complete elliptic integrals of the first and second kind, respectively.

In the limit $\lambda_{2} \rightarrow \lambda_{1}$ (i.e., $m \rightarrow 0$ ) we obtain

$$
\begin{aligned}
& v_{1}=v_{2}=2 \lambda_{1}+\frac{\left(\lambda_{4}-\lambda_{3}\right)^{2}}{2\left(\lambda_{3}+\lambda_{4}-2 \lambda_{1}\right)}, \\
& v_{3}=\frac{1}{2}\left(3 \lambda_{3}+\lambda_{4}\right), \quad v_{4}=\frac{1}{2}\left(\lambda_{3}+3 \lambda_{4}\right),
\end{aligned}
$$

and in another limit $m \rightarrow 0$, i.e. $\lambda_{3} \rightarrow \lambda_{4}$, we have

$$
\begin{aligned}
& v_{1}=\frac{1}{2}\left(3 \lambda_{1}+\lambda_{2}\right), \quad v_{2}=\frac{1}{2}\left(\lambda_{1}+3 \lambda_{2}\right), \\
& v_{3}=v_{4}=2 \lambda_{4}+\frac{\left(\lambda_{2}-\lambda_{1}\right)^{2}}{2\left(\lambda_{1}+\lambda_{2}-2 \lambda_{4}\right)} .
\end{aligned}
$$

Having received the basic equations, we can now proceed to the description of nonlinear trigonometric solutions for the KB system (1).

\section{TRIGONOMETRIC SHOCK WAVE}

Here we consider formation of the trigonometric shock wave pattern. In case of conventional initial conditions with uniform distributions on both sides of the initial step-like discontinuity such a structure is not generated [12. However, there exist quite natural initial conditions for which this kind of solutions does appear. Let the initial conditions have the following form. On the one side of the point $x=0$ we have constant distributions of $h$ and $u$, and on the other side there is a non-modulated nonlinear periodic wave described by four constant parameters $\lambda_{i}$. This means that we are interested in evolution of an initially sharp border of the wavy region. Before turning to oscillatory solutions, let us first consider the dispersionless case.

For smooth enough wave distributions we can neglect the last dispersion term in the first equation of the system (1) and arrive at the dispersionless equations

$$
h_{t}+(h u)_{x}=0, \quad u_{t}+u u_{x}+h_{x}=0,
$$




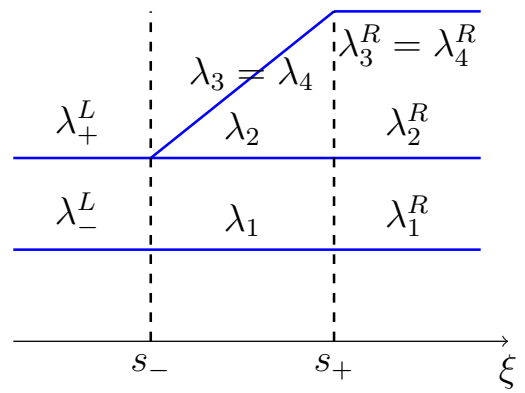

FIG. 1. Sketches of the behavior of the Riemann invariants in trigonometric dispersive shock wave solutions of the Whitham equations with $\lambda_{3}=\lambda_{4}$. Vertical dashed lines indicated by $s_{-}$and $s_{+}$define the edges of a trigonometric shock wave. Corresponding wave structure is shown in Fig. 2

which coincide with the well-known shallow water equations. First of all, this system admits a trivial solution for which $h=$ const and $u=$ const. We shall call such a solution a "plateau". We introduce the so-called Riemann invariants

$$
\lambda_{ \pm}=\frac{u}{2} \pm \sqrt{h}
$$

Using these dispersionless Riemann invariants, equations (33) can be written in the following diagonal form

$$
\frac{\partial \lambda_{ \pm}}{\partial t}+v_{ \pm}\left(\lambda_{-}, \lambda_{+}\right) \frac{\partial \lambda_{ \pm}}{\partial x}=0
$$

where

$$
v_{ \pm}\left(\lambda_{-}, \lambda_{+}\right)=\frac{1}{2}\left(3 \lambda_{ \pm}+\lambda_{\mp}\right) .
$$

These dispersionless variables are required for the correct choice of the initial state.

We assume that at the initial moment the profile is divided into two parts by the point $x=0$. We consider two types of profile. First, at $x<0$ there is a plateau characterized by the constant dispersionless invariants $\lambda_{-}=$const, $\lambda_{+}=$const, and for $x>0$ there is a nonmodulated wave described by the Eq 20$)$ (see Fig. 1 and left column in Fig. 2). We shall denote the parameters of this periodic wave as $\lambda_{1}^{R}, \lambda_{2}^{R}$, and $\lambda_{3}^{R}=\lambda_{4}^{R}$. The second type of initial state is similar: there is a non-modulated wave described by Eq 25 with $\lambda_{1}^{L}=\lambda_{2}^{L}=$ const, $\lambda_{3}^{L}=$ const, $\lambda_{4}^{L}=$ const at $x<0$ and a plateau with $\lambda_{-}=$const, $\lambda_{+}=$const at $x>0$ (see Fig. 3 and left column in Fig. 4 ).

We now turn to the study of situations when the dispersion effects are taken into account. In order to satisfy the matching conditions, the only possible solution arising from a given initial state may be a trigonometric shock wave. Trigonometric shock wave can be represented approximately as a modulated nonlinear periodic wave in which parameters $\lambda_{i}$ change slowly along the wave structure. In such a modulated periodic solution two equal Riemann invariants are changing and the other two remain constant along the entire shock. Thus, in our case the constant Riemann invariants and the Riemann invariants at the boundaries of the structure should have equal values. This situation resembles the so called 'contact discontinuity' which plays an important role in the theory of viscous shocks (see, e.g., [16]). This type of DSW was first reported in Ref. [17] where the evolution of a step problem was studied for the focusing modified KdV equation. In Ref. [18 these (trigonometric) DSWs were called contact DSWs. The trigonometric shock waves for the KB system are described by the modulated finiteamplitude nonlinear periodic solutions (20) or (25). The evolution of the trigonometric shock wave is determined by the Whitham equations (28). In our case of the steplike initial conditions we have to find self-similar solutions for which all Riemann invariants depend only on $\xi=x / t$, and the Whitham equations reduce to

$$
\frac{d \lambda_{i}}{d \xi} \cdot\left[v_{i}\left(\lambda_{1}, \lambda_{2}, \lambda_{3}, \lambda_{4}\right)-\xi\right]=0, \quad i=1,2,3,4 .
$$

If, for instance, we consider the first type of initial condition then in this situation, shown in Fig. 1] trigonometric shock wave has two equal parameters $\lambda_{3}=\lambda_{4}$ and the invariants $\lambda_{1}$ and $\lambda_{2}$ are constant along the whole wave pattern including shock region. We assume that there is a plateau with dispersionless Riemann $\lambda_{-}^{L}$ and $\lambda_{+}^{L}$ invariants to the left of the trigonometric shock wave. Thus we have $v_{3}\left(\lambda_{-}^{L}, \lambda_{+}^{L}, \lambda_{4}(\xi), \lambda_{4}(\xi)\right)=$ $v_{4}\left(\lambda_{-}^{L}, \lambda_{+}^{L}, \lambda_{4}(\xi), \lambda_{4}(\xi)\right)=\xi$. Consequently, we obtain

$$
\begin{aligned}
& \lambda_{1}=\lambda_{-}^{L}, \quad \lambda_{2}=\lambda_{+}^{L}, \\
& v_{4}=2 \lambda_{4}-\frac{\left(\lambda_{+}^{L}-\lambda_{-}^{L}\right)^{2}}{\lambda_{+}^{L}+\lambda_{-}^{L}-2 \lambda_{4}}=\xi,
\end{aligned}
$$

where the last formula determines the dependence of $\lambda_{4}$ on $\xi$, which can be represented in the explicit form

$$
\begin{aligned}
\lambda_{4}(\xi)=\frac{1}{4}\left[\xi+\lambda_{+}^{L}+\lambda_{-}^{L}+\right. \\
\left.\sqrt{\left(\xi-\lambda_{+}^{L}-\lambda_{-}^{L}\right)^{2}+2\left(\lambda_{+}^{L}-\lambda_{-}^{L}\right)^{2}}\right] .
\end{aligned}
$$

Here $\xi$ varies within the interval $s_{-} \leq \xi \leq s_{+}$with

$$
s_{-}=\frac{3 \lambda_{+}^{L}+\lambda_{-}^{L}}{2}, \quad s_{+}=2 \lambda_{4}^{R}+\frac{\left(\lambda_{+}^{L}-\lambda_{-}^{L}\right)^{2}}{2\left(\lambda_{+}^{L}+\lambda_{-}^{L}-2\right)},
$$

where $\lambda_{4}^{R}=\lambda_{3}^{R}$ is the maximum value of $\lambda_{4}(\xi)$ defined by the right boundary condition of trigonometric shock wave. The wavelength in this case is given by the formula

$$
L=\frac{2 \pi}{\sqrt{\left(\lambda_{4}(\xi)-\lambda_{-}^{L}\right)\left(\lambda_{4}(\xi)-\lambda_{+}^{L}\right)}} .
$$

Substitution of $\lambda_{i}$ into Eq. 20 with subsequent substitution into Eq. 12 yields the modulated periodic solutions resulting in the trigonometric shock wave structure. 


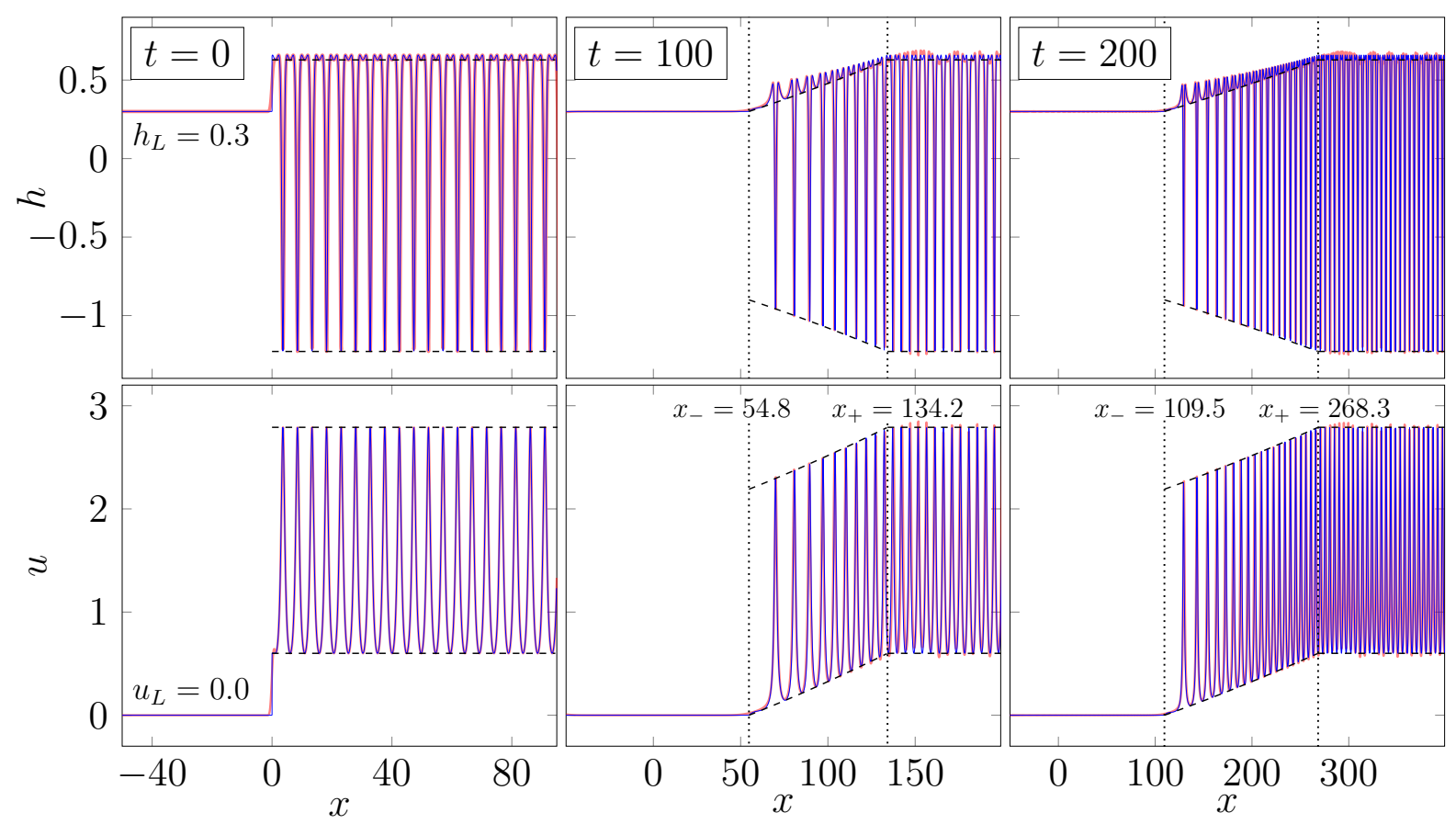

FIG. 2. Evolution of a trigonometric shock wave for $h_{L}=0.3, u_{L}=0$ and $\lambda_{3}^{R}=\lambda_{4}^{R}=0.85$. Figures show the initial state (left column) and wave profiles for depth $h$ and flow velocity $u$ for $t=100$ (middle column) and $t=200$ (right column). Red (thick) curves show the result of numerical calculations, and a blue (thin) one shows the analytical solution. Dashed lines illustrate envelopes of wave structure, vertical dashed lines indicate the edges of the trigonometric shock wave $\left(x_{-}\right.$and $\left.x_{+}\right)$. We have here dark solitons of elevation $h$ and bright solitons of flow velocity $u$ at the soliton edge of the shock. The corresponding diagram of Riemann invariants is shown in Fig. 1 .

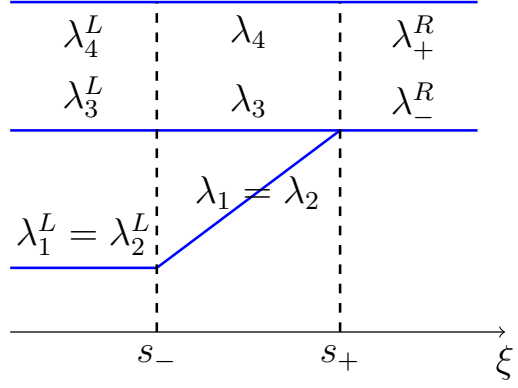

FIG. 3. Sketches of the behavior of the Riemann invariants in trigonometric dispersive shock wave solutions of the Whitham equations with $\lambda_{1}=\lambda_{2}$. Vertical dashed lines indicated by $s_{-}$and $s_{+}$define the edges of a trigonometric shock wave. Corresponding wave structure is shown in Fig. 4

Comparison of the obtained analytical solution with numerical calculations is shown in Fig. 2 for different values of time $t$. One can see that the trigonometric shock wave is located between the edges with coordinates $x_{-}=s_{-} t$ and $x_{+}=s_{+} t$. This wave matches at its left edge with the left plateau and at its right edge with the non-modulated wave.
In a similar way, we can consider the second type of initial conditions. For this case the trigonometric shock wave has $\lambda_{1}=\lambda_{2}$. An example of the diagram of Riemann invariants is shown in Fig. 3 . Obviously, we get a symmetric situation, where the trigonometric shock wave matches the plateau characterised by the values $\lambda_{-}^{R}$ and $\lambda_{+}^{R}$ of the Riemann invariants at the right edge and the non-modulated wave with the values $\lambda_{1}^{L}=\lambda_{2}^{L}=$ const, $\lambda_{3}^{L}=\lambda_{-}^{R}$ and $\lambda_{2}^{L}=\lambda_{+}^{R}$ of the Riemann invariants of the Whitham system at the left edge. The solution of the Whitham equations takes the form

$$
\begin{aligned}
& v_{1}=v_{2}=2 \lambda_{1}+\frac{\left(\lambda_{+}^{R}-\lambda_{-}^{R}\right)^{2}}{2\left(\lambda_{+}^{R}+\lambda_{-}^{R}-2 \lambda_{1}\right)}=\xi, \\
& \lambda_{3}=\lambda_{-}^{R}=\lambda_{-}^{R}, \quad \lambda_{4}=\lambda_{+}^{R}=\lambda_{+}^{R},
\end{aligned}
$$

or

$$
\begin{aligned}
\lambda_{1}(\xi)=\frac{1}{4} & {\left[\xi+\lambda_{+}^{R}+\lambda_{-}^{R}-\right.} \\
& \left.\sqrt{\left(\xi-\lambda_{+}^{R}-\lambda_{-}^{R}\right)^{2}+2\left(\lambda_{+}^{R}-\lambda_{-}^{R}\right)^{2}}\right]
\end{aligned}
$$

where $\xi$ varies in the interval $s_{-} \leq \xi \leq s_{+}$with

$$
s_{-}=-2 \lambda_{1}^{L}+\frac{\left(\lambda_{+}^{R}-\lambda_{-}^{R}\right)^{2}}{2\left(\lambda_{+}^{R}+\lambda_{-}^{R}+2\right)}, \quad s_{+}=\frac{\lambda_{+}^{R}+3 \lambda_{-}^{R}}{2} .
$$




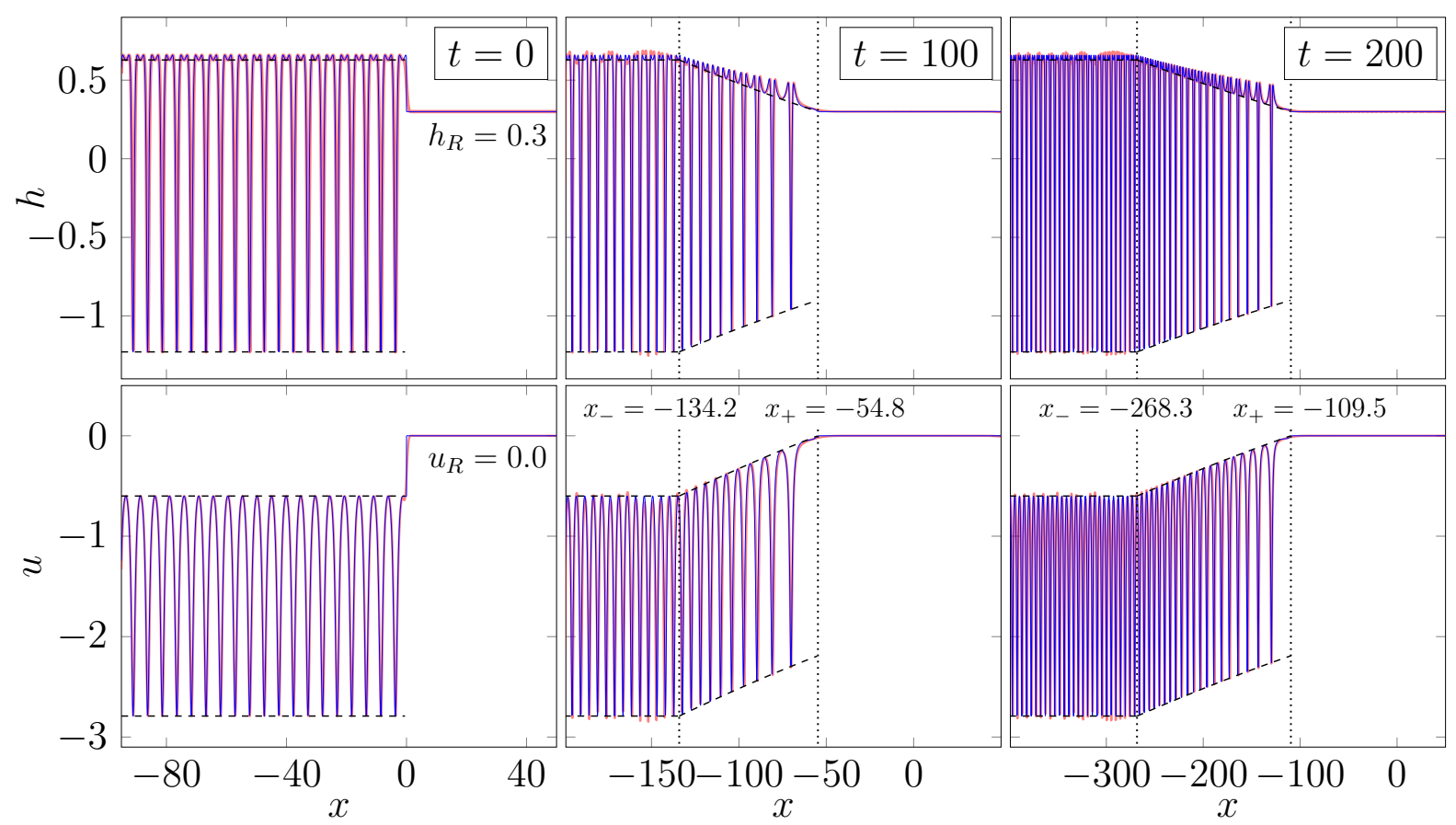

FIG. 4. Evolution of a trigonometric shock wave $h_{R}=0.3, u_{R}=0$ and $\lambda_{1}^{L}=\lambda_{2}^{L}=-0.85$. Figures show the initial state (left column) and wave profiles for depth $h$ and flow velocity $u$ for $t=100$ (middle column) and $t=200$ (right column). Red (thick) curves show the result of numerical calculations, and a blue (thin) one shows the analytical solution. Dashed lines illustrate envelopes of wave structure, vertical dashed lines indicate the edges of the trigonometric shock wave $\left(x_{-}\right.$and $\left.x_{+}\right)$. On the contrary to the case of Fig. 2 now we get dark solitons of both elevation $h$ and flow velocity $u$ at the soliton edge of the shock. The corresponding diagram of Riemann invariants is shown in Fig. 3 .

Parameter $\lambda_{1}^{L}=\lambda_{2}^{L}$ is determined again by the initial conditions at the boundary with the non-modulated wave. The wavelength is given here by the formula

$$
L=\frac{2 \pi}{\sqrt{\left(\lambda_{1}(\xi)-\lambda_{-}^{R}\right)\left(\lambda_{1}(\xi)-\lambda_{+}^{R}\right)}} .
$$

Our analytical results and numerical simulations for the corresponding wave structures are compared in Fig. 4. Now trigonometric shock wave is located between the edge points $x_{-}=s_{-} t$ and $x_{+}=s_{+} t$ indicated by dotted lines. One can see that numerical calculations (red thick) agree with the analytical curve (blue thin) very well.

Although the wave patterns look similar, one should notice that in Fig. 2 the distribution of $u(x, t)$ tends to bright solitons at the soliton edge of the shock whereas in Fig. 4 we get dark solitons of $u(x, t)$ at this edge. In both cases the variable $h(x, t)$ takes negative values and that limits application of the developed theory to water waves. Nevertheless it is applicable to other physical situations such as nonlinear shocks in two-component Bose-Einstein condensates or in magnetics.

\section{CONCLUSION}

In this Letter we have considered the cases in which the trigonometric shock waves arise when their evolution is governed by the integrable Kaup-Boussinesq system. The initial state contains a nonlinear wave which significantly extends the set of wave patterns which can be generated in various physical situations. Our results can find applications as approximation to the dynamics of polarization waves in two-component Bose-Einstein condensates and in magnetic systems with easy-plane anisotropy which are not constrained by the condition that $h$ must be positive.

\section{ACKNOWLEDGMENTS}

This work was supported by a grant from Foundation for the Advancement of Theoretical Physics and Mathematics "BASIS".
[1] A. V. Gurevich and L. P. Pitaevskii, Zh. Eksp. Teor. Fiz. 65, 590 (1973) [Sov. Phys. JETP 38, 291 (1974)].
[2] G. B. Whitham, Proc. Roy. Soc. London, 283, 238 (1965). 
[3] G. A. El, M. A. Hoefer, Physica D 333, 11-65 (2016).

[4] A. M. Kamchatnov, Physics-Uspekhi 64, 48 (2021).

[5] D. E. Korteweg, G. de Vries Phil. Mag. 39, 422443 (1895).

[6] B. Benjamin, M. J. Lighthill, Proc. Roy. Soc. Lond., 224, 448 (1954).

[7] J.Boussinesq, Mém. Prés. Div. Sav. Acad. Sci. Inst. Fr., 23, 1 (1877).

[8] D. J. Kaup Progr. Theor. Phys., 54, 2, 396-408 (1975).

[9] V. B. Matveev and M. I. Yavor, Ann. Inst. Henri. Poincaré A, 31, 25 (1979).

[10] G. A. El, R. H. J. Grimshaw, and M. V. Pavlov Stud. Appl. Math. 106, 157 (2001).

[11] G. A. El, R. H. J. Grimshaw, and A. M. Kamchatnov Stud. Appl. Math. 114, 395 (2005).
[12] T. Congy, S. K. Ivanov, A. M. Kamchatnov, N. Pavloff Chaos 27, 083107 (2017).

[13] S. K. Ivanov, A. M. Kamchatnov, T. Congy, N. Pavloff Phys. Rev. E 96, 062202 (2017).

[14] A. M. Kamchatnov, Nonlinear Periodic Waves and Their Modulations - An Introductory Course (World Scientific, Singapore, 2000).

[15] M. Abramowitz and I. A. Stegun, Handbook of mathematical functions, (Dover Publications, New-York, 1972).

[16] L. D. Landau and E. M. Lifshitz, Fluid Mechanics, Pergamon, Oxford, (1959).

[17] T. R. Marchant, Wave Motion, 45, 540-555 (2008).

[18] G. A. El, M. A. Hoefer, and M. Shearer, SIAM Review 59, 3 (2017). 